\section{Professional issues when treating pets of owners of no fixed abode}

\section{Ruth Serlin}

In our usual veterinary lives, we have to make many different professional decisions each and every day. Familiarity with our environment, clients, colleagues and codes of conduct means that most of our decisions are made before we are even aware of the different elements and nuances of the process. It maybe there are local practice-based guidelines or rules that are straightforward to follow; alternatively, discussion with a more experienced colleague will help when we are finding it hard to chose between one course of action and one or several others.

In our experience in setting up and running volunteer-led initiatives treating pets owned by homeless and vulnerably housed people, our decision-making process can become challenged and tested. The problems we encounter are both complicated and complex, and as veterinary professionals, we are often far out of our familiar comfort zone of the veterinary clinic.

In this interactive session, we will firstly discuss the need for volunteer-led initiatives to comply with the legal and ethical requirements of veterinary practice and give some advice on ensuring compliance with the RVCS and Veterinary Medicines Directorate. Then we will move into exploring the challenges to professionalism that we frequently encounter. These challenges relate to: having a shifting client base; clients with their own complex needs; the heightened human-animal bond between our clients and their pet; the need to interact with multiple agencies; safeguarding issues and the very public nature of our work

We will do this by presenting and discussing vignettes that relate to real-life situations we have faced and managed. As part of this process, we will introduce you to a professional decision-making framework and process that will give you confidence to balance the conflicting needs of different stakeholders and then be able to address your roles and responsibilities.

\section{KEY LEARNING OBJECTIVES}

- Recognize the need for legal and ethical compliance in volunteer-led initiatives treating pets owned by homeless and vulnerably housed people

- Approach complex situations related to treating these pets by listing the various stakeholders concerned and weighing up the various needs of the stakeholders

- Be able to come to a decision, balancing out the needs and requirements of all the stakeholders whilst upholding professional responsibilites

\section{Practical considerations when treating pets of owners of no fixed abode: the veterinary team as inclusion medicine allied health professionals}

\section{Gabriel Galea}

There are myriad causes of homelessness. Adverse childhood experiences (e.g. sexual abuse, domestic violence, being expelled from school) are internationally recognized as substantial risk factors for adult homelessness, together with prior traumatic brain injury, autism and having a minority gender identity. In a UK population, $37.4 \%$ of individuals who experienced homelessness were identified to have probable psychotic or neurotic disorders, compared with $25.4 \%$ who primarily had alcohol or drug dependencies. Recent investigative journalism work identified 11 homeless deaths per week in the UK, with a large proportion of deaths being caused by preventable or treatable conditions such as tuberculosis, pneumonia and gastric ulcers. These findings paint a picture of homelessness as both a cause and consequence of marginalization and social exclusion.

When an individual or family lose their home they are in many cases offered emergency accommodation by local councils, who are responsible for social service provision. Most temporary accommodation is not pet friendly, causing the majority of owners to give up their pets at an already traumatic period in their lives. Many are unable to part with their companions, forcing themselves to sleep rough in order to keep their pets. Irrespective of whether the pet is a long-term family member or newly acquired, caring for a pet while homeless carries tremendous costs. Tangible costs include reduced mobility, vulnerability to bereavement, and exclusion from health and housing services which do not have proactive pet policies. It is therefore not surprising that individuals who make these sacrifices to keep a pet while homeless score higher on pet attachment and empathy scores than owners in secure accommodation. Equally, the benefits of pet ownership are as evident for individuals experiencing homelessness as they are for those in secure housing. In particular, homeless pet owners report their pets give them resilience, a sense of redemption, and motivation to reduce substance misuse. 